\title{
Molecular viewpoint on the crystal growth dynamics driven by solution flow
}

\author{
D. Maes ${ }^{\dagger}$ and James F. Lutsko*,+ \\ $\dagger$ Structural Biology Brussels, Vrije Universiteit Brussel, Pleinlaan 2, 1050, Brussels, \\ Belgium \\ $\ddagger$ Center for Nonlinear Phenomena and Complex Systems, Code Postal 231, Université \\ Libre de Bruxelles, Boulevard du Triomphe, 1050 Brussels, Belgium \\ E-mail: jlutsko@ulb.ac.be
}

\section{Multiscale analysis of growth rate}

\section{Model}

A simple analysis for the interaction of diffusion and growth in our model is possible under the assumption of planar symmetry. Specifically, we assume that diffusion is fast compared to step growth so that inhomogeneities in the density in the planes parallel to the crystal surface are very small or are short-lived compared to the time-scale of step growth. In this case, the concentration depends only on the $z$-coordinate, $c_{t}(z)$, and satisfies a diffusion equation

$$
\frac{\partial c_{t}(z)}{\partial t}=D \frac{\partial^{2} c_{t}(z)}{\partial z^{2}}
$$

Boundary conditions are needed at the top and bottom of the simulation cell. Consider one cell in the computational lattice in the top-most layer of the fluid. The average number of molecules expected in such a cell is $c_{t}\left(L_{z}\right) a^{3}$. Molecules can enter and leave via any of 
the six faces of the cell but movement via the lateral faces is of no importance since we are assuming homogeneity within the plane: this means that the rates of molecules entering and leaving via the lateral faces exactly balance. The rate at which molecules leave via the top and bottom faces is simply $\nu_{\text {jump }} c_{t}\left(L_{z}\right) a^{3}$ in each case. The rate at which molecules enter at the bottom is correspondingly $\nu_{\text {jump }} c_{t}\left(L_{z}-a\right) a^{3}$ while the rate at which they enter via the top is the product of the rate at which insertions are attempted and the probability that the cell is empty, $r_{\text {add }}\left(1-c_{t}\left(L_{z}\right) a^{3}\right)$. Putting this together,

$$
\begin{aligned}
\frac{\partial a^{3} c_{t}\left(L_{z}\right)}{\partial t} & =\left(r_{\text {add }}\left(1-c_{t}\left(L_{z}\right) a^{3}\right)-\nu_{\text {jump }} c_{t}\left(L_{z}\right) a^{3}\right) \\
& +\left(\nu_{\text {jump }} c_{t}\left(L_{z}-a\right) a^{3}-\nu_{\text {jump }} c_{t}\left(L_{z}\right) a^{3}\right)
\end{aligned}
$$

or in the continuum limit,

$$
\frac{\partial c_{t}\left(L_{z}\right)}{\partial t} \simeq a^{-3} r_{\mathrm{add}}-\left(r_{\mathrm{add}}+\nu_{\mathrm{jump}}\right) c_{t}\left(L_{z}\right)-a^{-1} D\left(\frac{\partial c_{t}(z)}{\partial z}\right)_{z=L}
$$

At the interface between the crystal and the fluid, taken to be at position $z=H_{t}$, there must be a balance between the rate at which material arrives at the surface, via diffusion, and the rate at which the crystal grows giving

$$
-D\left(\frac{\partial c_{t}(z)}{\partial z}\right)_{H_{t}} L_{x} L_{y}=a^{-2} v\left(c_{t}\left(H_{t}\right)\right) L_{y}
$$

where it is assumed that the steps are parallel to the $y$-direction and grow in the $x$-direction. The crystal increases height by one lattice cell, so $H_{t} \rightarrow H_{t}+a$, when a total of $N_{x} N_{y}$ molecules have been added so, in the continuum limit, we have that $d H=\frac{a}{N_{x} N_{y}} d M$ where $d M$ is the change in mass of the crystal. As a step grows a distance $d L_{x}=v d t$, the crystal adds $L_{y} d L_{x} / a^{2}$ molecules so $d M / d t=L_{y} v / a^{2}$ and the balance requires

$$
\frac{d H_{t}}{d t}=\frac{a}{N_{x} N_{y}} \frac{L_{y} v\left(c_{t}\left(H_{t}\right)\right)}{a^{2}}=\frac{a}{L_{x}} v\left(c_{t}\left(H_{t}\right)\right)
$$


So, the model for the rate of step growth can be written as:

$$
\begin{aligned}
\frac{\partial c_{t}(z)}{\partial t} & =D \frac{\partial^{2} c_{t}(z)}{\partial z^{2}}, c_{t}\left(L_{z}\right)=A_{t} \text { and } c_{t}\left(H_{t}\right)=B_{t} \\
\frac{d H_{t}}{d t} & =\frac{a}{L_{x}} v\left(B_{t}\right) \\
\frac{d A_{t}}{d t} & =a^{-3} r_{\text {add }}-\left(r_{\text {add }}+\nu_{\text {jump }}\right) A_{t}-a^{-1} D\left(\frac{\partial c_{t}(z)}{\partial z}\right)_{z=L} \\
v\left(B_{t}\right) & =-a^{2} L_{x} D\left(\frac{\partial c_{t}(z)}{\partial z}\right)_{z=H_{t}}
\end{aligned}
$$

\section{Quasi-steady state}

The problem admits of a natural small parameter, $\epsilon \equiv \frac{a}{L_{x}}$. This arises because the rate of growth in the $z$-direction is necessarily small compared to the rate of step advancement. We can therefore use this to construct a simple multiscale analysis. We slightly rewrite the system of equations as

$$
\begin{aligned}
\frac{\partial c_{t}(z)}{\partial t} & =D \frac{\partial^{2} c_{t}(z)}{\partial z^{2}}, c_{t}\left(L_{z} ; H_{t}\right)=A_{t}\left(H_{t}\right) \text { and } c_{t}\left(H_{t} ; H_{t}\right)=B_{t}\left(H_{t}\right) \\
\frac{d H_{t}}{d t} & =\epsilon v\left(B_{t}\right) \\
\frac{d A_{t}}{d t} & =a^{-3} r_{\text {add }}-\left(r_{\text {add }}+\nu_{\text {jump }}\right) A_{t}-\epsilon a^{-2} D \frac{L_{x}}{L_{z}}\left(L_{z} \frac{\partial c_{t}(z)}{\partial z}\right)_{z=L} \\
v\left(B_{t}\right) & =-a^{2} \frac{L_{x}}{L_{z}} D\left(L_{z} \frac{\partial c_{t}(z)}{\partial z}\right)_{z=H_{t}}
\end{aligned}
$$

to emphasize the fact that we expect the concentration near the crystal to be close to its

equilibrium value so that $\frac{\partial c_{t}(z)}{\partial z} \sim \frac{c\left(L_{z}\right)-c_{e q}}{L_{z}}$. Hence, we wish to treat $L_{z} \frac{\partial c_{t}(z)}{\partial z}$ as being of order $\epsilon^{0}$. With this in mind, it is clear that the only consequence of $\epsilon$ being small is that the rate of growth in the $z$-direction is small. We could use this as the basis for a general multiscale analysis but here we restrict attention to the quasi-steady state in which the only time-dependence of the concentration occurs via its dependence on $H_{t}$. In this case, we have 
that $c_{t}(z) \rightarrow c\left(z ; H_{t}\right)$ and

$$
\frac{\partial c_{t}(z)}{\partial t}=\frac{\partial c\left(z ; H_{t}\right)}{\partial H_{t}} \frac{d H_{t}}{d t}=\epsilon v\left(B\left(H_{t}\right)\right) \frac{\partial c\left(z ; H_{t}\right)}{\partial H_{t}}
$$

SO

$$
\begin{aligned}
\epsilon v\left(B\left(H_{t}\right)\right) \frac{\partial c\left(z ; H_{t}\right)}{\partial H_{t}} & =D \frac{\partial^{2} c\left(z ; H_{t}\right)}{\partial z^{2}}, c\left(L_{z} ; H_{t}\right)=A\left(H_{t}\right) \text { and } c\left(H_{t} ; H_{t}\right)=B\left(H_{t}\right) \\
\epsilon v\left(B\left(H_{t}\right)\right) \frac{d A\left(H_{t}\right)}{d H_{t}} & =a^{-3} r_{\text {add }}-\left(r_{\text {add }}+\nu_{\text {jump }}\right) A\left(H_{t}\right)-\epsilon a^{-2} D \frac{L_{x}}{L_{z}}\left(L_{z} \frac{\partial c\left(z ; H_{t}\right)}{\partial z}\right)_{z=L} \\
v\left(B\left(H_{t}\right)\right) & =-a^{2} \frac{L_{x}}{L_{z}} D\left(L_{z} \frac{\partial c\left(z ; H_{t}\right)}{\partial z}\right)_{z=H_{t}}
\end{aligned}
$$

with the height of the crystal being determined by $\frac{d H_{t}}{d t}=\epsilon v\left(B_{t}\right)$. These equations can be solved perturbatively by expanding $c\left(z ; H_{t}\right)=c^{(0)}\left(z ; H_{t}\right)+\epsilon c^{(1)}\left(z ; H_{t}\right)+\ldots$ and solving order by order in $\epsilon$. At lowest order, this gives

$$
\begin{aligned}
0 & =D \frac{\partial^{2} c^{(0)}\left(z ; H_{t}\right)}{\partial z^{2}} \\
0 & =a^{-3} r_{\text {add }}-\left(r_{\text {add }}+\nu_{\text {jump }}\right) A^{(0)}\left(H_{t}\right) \\
v\left(B^{(0)}\left(H_{t}\right)\right) & =-a^{2} \frac{L_{x}}{L_{z}} D\left(L_{z} \frac{\partial c^{(0)}\left(z ; H_{t}\right)}{\partial z}\right)_{z=H_{t}}
\end{aligned}
$$

with solution

$$
\begin{aligned}
c^{(0)}\left(z ; H_{t}\right) & =A^{(0)}+\left(B^{(0)}\left(H_{t}\right)-A^{(0)}\right) \frac{z-L_{z}}{H_{t}-L_{z}} \\
A^{(0)} & =\frac{a^{-3} r_{\text {add }}}{r_{\text {add }}+\nu_{\text {jump }}} \\
v\left(B^{(0)}\left(H_{t}\right)\right) & =-a^{2} D\left(B^{(0)}\left(H_{t}\right)-A^{(0)}\right) \frac{L_{x}}{H_{t}-L_{z}}
\end{aligned}
$$

which is the result used in the main text. Notice that the growth rate is zero when $B^{(0)}\left(H_{t}\right)=$ $c^{(0)}\left(H_{t} ; H_{t}\right)$ is the equilibrium concentration. In this case, there can be no gradients in the density so it must also be the case that $B^{(0)}\left(H_{t}\right)=A^{(0)}$ which identifies $c_{\text {eq }}=\frac{a^{-3} r_{\text {add }}}{r_{\text {add }}+\nu_{\text {jump }}}$ as 
quoted in the main text. The solution could be continued to higher order with no difficulty.

As stated at the beginning of this Appendix, these calculations are only valid if inhomogeneities in the planes parallel to the crystal surface are either very small or are short-lived on the timescale of step growth. The fact that our results are in good agreement with the simulations supports this assumption under the conditions considered here. We have, in selected cases, actually determined the variation of the density in the fluid plane immediately above the crystal surface, as a function of position. This requires tracking the step front and averaging in a co-moving frame and so introduces some uncertainties. Nevertheless, we see a clear minimum at the step face but it is quite small - only about $2 \%$ of the density far from the step face - which perhaps accounts for the good agreement we find between the simple model given here and the simulations.

\section{Mathematical symbols used in main text}

$a$ : the lattice spacing.

$\beta=1 / k_{B} T:$ the inverse temperature.

$c_{\text {eq }}$ : equilibrium concentration in the fluid for a given chemical potential (i.e. with no crystal present).

$c_{0}$ : the concentration at the crystal surface.

$c_{\text {coex }}$ : fluid concentration at coexistence with the crystal.

$c(z ; t)$ : fluid concentration averaged over $x-y$ planes.

$c^{*}=c a^{3}$ : dimensionless concentration.

$c$ : advection velocity.

$\chi\left(l ; \widehat{\mathbf{e}}_{\alpha}\right)$ : characteristic function for molecule $l$ in solution: it is equal to one if the neighbor in the direction of $\widehat{\mathbf{e}}_{\alpha}$ is unoccupied and zero otherwise.

$D$ : the low-density diffusion constant of the crystal-forming species in solution. $\mathcal{E}$ : any of the possible elementary events in the kMC simulation. 
$\varepsilon$ : absolute value of nearest-neighbor bond energy

$\widehat{\mathbf{e}}_{\alpha}$ : unit vector in one of the cartesian directions $(\alpha= \pm x, \pm y$ or $\pm z)$.

$E_{i j}$ : energy of surface molecule at position $(i, j)$.

$E(\mathbf{S})$ : energy of state $\mathbf{S}$.

$E_{c}(\mathbf{S})$ : Energy of crystal in state $\mathbf{S}$.

$\gamma$ : liquid-crystal surface tension.

$H_{t}$ : average height of crystal at time $t$.

$\mathbf{H}$ : Array giving the height of the crystal at each $x, y$ lattice site.

$\mu$ : chemical potential of crystal-forming species in solution.

$\mu_{\mathrm{eq}}$ : Chemical potential giving equilibrium with the crystal.

$\Delta \mu_{\text {eff }}$ effective supersaturation at the crystal surface.

$\Delta \mu^{*}$ : dimensionless chemical potential.

$\Delta \mu=\mu-\mu_{\mathrm{eq}}$ : supersaturation applied at the crystal surface (SOS model) or at the upper boundary (our model).

$M(t)$ : mass of the crystal at time $t$.

$n_{t}(\mathbf{s})$ : number of molecules in the fluid at site $\mathbf{s}$.

$n_{i j}$ : number of nearest neighbor bonds of surface molecule at site $(i, j)$.

$\mathcal{N}$ : total number of possible elementary events in the kMC algorithm

$N_{\text {Fluid }}(t)$ : in the kMC model, the number of molecules in the fluid at time $t$.

$N_{x}$ : Number of lattice sites in the x-direction (direction of step growth)

$N_{y}$ : Number of lattice sites in the y-direction (parallel to step face) b $N_{z}$ : Number of lattice sites in the z-direction (direction perpindicular to crystal surface)

$N$ : the number of crystal molecules in solution

$\nu_{\text {jump }}$ : attempt frequency of attempted jumps of molecule in solution.

$\nu_{\text {add }}$ : attempt frequency of molecules entering via the upper boundary.

$\nu_{\text {detach }}$ : attempt frequency of molecules detaching from surface.

$\nu^{*}=\nu / r_{0}$ : dimensionless frequency. 
$\nu_{0}$ : the attempt frequency.

$q$ : parameter characterizing the bias of the jump probabilities to simulate flow.

$r_{i j}^{+}$: rate of attachment of molecules at surface site $(i, j)$.

$r_{i j}^{-}$: rate of detachment of molecules at surface site $(i, j)$.

$r_{0}$ : constant used to map rates to probabilities.

$r_{\text {jump }}$ : rate of jumps of molecules in solution.

$r_{\text {add }}\left(i, j, N_{z}\right)$ : rate of molecules entering via the upper boundary at site $(i, j)$.

$r_{\text {detach }}\left(i, j, H_{i j}\right)$ : rate of crystal surface molecule at site $(i, j)$ detaching from surface.

$r\left(\mathbf{S} \rightarrow \mathbf{S}^{\prime}\right)$ : rate of transition from state $\mathbf{S}$ to state $\mathbf{S}^{\prime}$.

$r(\mathcal{E})$ : rate of event $\mathcal{E}$ occuring.

$\mathbf{s}(i, j, k)$ : a site in the fluid with coordinates $i, j, k$.

$\mathbf{S}=(\mathbf{H}, N)$ : specification of the state of the system.

$T$ : the temperature

$\delta t$ : the time step.

$T^{*}=k_{B} T / \varepsilon$ : dimensionless temperature.

$v_{1}, v_{2}$ : constants used to fit step veloctiy to superaturation.

$v(c)$ : step velocity as a function of concentration.

$v$ : step velocity. 